COMMUNICATIONS IN

ANALYSIS AND GEOMETRY

Volume 4, Number 2, 261-284, 1996

\title{
On conformally flat submanifolds
}

\author{
MARCoS DAJCZER \& LUIS A. FloRIT
}

\section{Introduction.}

An immersed submanifold $f: M^{n} \rightarrow \mathbf{R}^{n+p}$ in standard flat Euclidean space, endowed with the induced metric, is said to be conformally flat if each point has a neighborhood conformal to $\mathbf{R}^{n}$. Around 1919, nonflat conformally flat hypersurfaces $(p=1, n \geq 4)$ were completely described by E. Cartan $\left(\left[\mathbf{C a}_{2}\right]\right)$ as being any envelope of a 1-parameter family of spheres. In this case, the geometric parametric description (see $[\mathbf{C Y}],[\mathbf{C D M}],\left[\mathbf{A D}_{2}\right]$ or [Da]) is an immediate consequence of the existence, at any point, of a principal curvature of multiplicity at least $n-1$.

For higher but still low codimension, namely, $p \leq n-3$, the algebraic structure of the second fundamental form of a conformally flat submanifold has also been well understood for some time due to the work of Moore $\left(\left[\mathbf{M o}_{1}\right]\right.$; see also $\left.[\mathbf{M M}]\right)$. His extension of Cartan's result implies that, generically, $M^{n}$ carries a $(n-p)$-dimensional foliation by extrinsic spheres which immerse in $\mathbf{R}^{n+p}$ as round spheres.

It is well known ([ $\left.\left.\mathbf{A D}_{1}\right],[\mathbf{D a}]\right)$ that a simply connected Riemannian manifold $M^{n}, n \geq 3$, is conformally flat if and only if it can be realized as a hypersurface of the light cone $\mathbf{V}^{n+1}$ of the standard flat Lorentzian space $\mathbf{L}^{n+2}$. Recall that

$$
\mathbf{V}^{n+1}=\left\{X \in \mathbf{L}^{n+2}:\langle X, X\rangle=0, X \neq 0\right\} .
$$

Hence, in order to obtain examples of conformally flat submanifolds $M^{n}$ of $\mathbf{R}^{n+p}$, it suffices to produce a Riemannian manifold $N^{n+1}$ which admits isometric immersions $F: N^{n+1} \rightarrow \mathbf{R}^{n+p}$ and $G: N^{n+1} \rightarrow \mathbf{L}^{n+2}$ and then take $M^{n}$ as the intersection $G\left(N^{n+1}\right) \cap \mathrm{V}^{n+1}$.

Our first main result is that, for $p \leq n-3$, the above procedure generates all simply connected examples. In particular, Moore's spherical foliation is nothing else but the intersection with $\mathbf{V}^{n+1}$ of the at least $(n-p+1)-$ dimensional relative nullity foliation common to $F$ and $G$. 
The remaining part of the paper is devoted to the local classification of all conformally flat submanifolds in codimension $p=2$. In fact, this goal is achieved by two different means. Our first approach consists in putting together the above result with a description of all Riemannian manifolds $N^{m}$ which can be realized, simultaneously, as hypersurfaces in $\mathbf{R}^{m+1}$ and $\mathbf{L}^{m+1}$.

This last result is of independent interest and has other consequences. On one hand, it allows us to construct explicit counterexamples to the claims in $\left[\mathrm{No}_{1}\right]$ and $\left[\mathrm{No}_{2}\right]$. More importantly, it reveals a completely unexpected strong relation with the classical Sbrana-Cartan theory ([Sb], $\left[\mathbf{C a}_{1}\right]$, [DFT]) of isometrically deformable Euclidean hypersurfaces. It turns out that in order to admit an isometric immersion in $\mathbf{L}^{m+1}$, a nonflat hypersurface $N^{m}$ of $\mathbf{R}^{m+1}$ is either in one of three (out of five) classes of deformable Sbrana-Cartan hypersurfaces or has a similar structure as the elements of a fourth class. As a consequence, in codimension 2, we show that any 'generic' conformally flat submanifold either has as many isometric deformations as a certain surface in $\mathbf{R}^{3}$ or in the sphere $\mathbf{S}^{3}$, admits precisely a 1 -parameter family of deformations, or is isometrically rigid.

Making use of our previous results, we are then able to describe all nonflat codimension 2 conformally flat submanifolds in a parametric form. This second approach turns out to be much more involved than Cartan's description because, aside from hypersurfaces, the existence alone of a spherical foliation as above is far from sufficient to conclude conformal flatness.

Our parametrization and an observation due to Cartan then enable us to explicitly construct a large family of what seems to be the first known generic examples. Finally, in order to complete our classification, we extend the parametrization of flat surfaces in $\mathbf{R}^{4}$ obtained in [CD] to flat $n$-dimensional submanifolds of $\mathbf{R}^{n+2}$.

\section{The general case.}

We first recall some basic facts and definitions. Given an isometric immersion $F: N^{m} \rightarrow \mathbf{R}^{m+p}$, the relative nullity subspace $\Delta(x) \subset T_{x} N$ at $x \in N^{m}$ is defined as

$$
\Delta(x)=\left\{X \in T_{x} N: \alpha_{F}(X, Y)=0, \forall Y \in T_{x} N\right\},
$$

where $\alpha_{F}: T N \times T N \rightarrow T_{F}^{\perp} N$ stands for the vector valued second fundamental form. It is a standard fact that on any open subset where the index of relative nullity $\nu_{F}(x):=\operatorname{dim} \Delta(x)$ is constant, the relative nullity distribution is integrable and its leaves are totally geodesic in $N^{m}$ and $\mathbf{R}^{m+p}$. 
Consider a hypersurface $F: N^{m} \rightarrow \mathbf{R}^{m+1}$ with constant index $\nu_{F}=\ell$, $0 \leq \ell \leq m-1$. In this situation, we may locally parametrize $F$ by means of the Gauss parametrization which we briefly describe next for later use and refer to $\left[\mathbf{D G}_{1}\right]$ for further details.

Let $V^{m-\ell}$ be the quotient space of relative nullity leaves in an open subset $U \subset N^{m}$ with projection $\pi: U \rightarrow V^{m-\ell}$. The Gauss map $\xi: U \rightarrow \mathbf{S}^{m}(1)$ induces an isometric (with the induced metric) immersion $h: V^{m-\ell} \rightarrow$ $\mathbf{S}^{m}(1)$ so that $h \circ \pi=\xi$. Let $\mathcal{N}$ denote the normal bundle of $h$ in $\mathbf{S}^{m}(1) \subset$ $\mathbf{R}^{m+1}$ and let $\gamma$ be the "support function" defined by $\gamma \circ \pi=\langle F, \xi\rangle$. The Gauss parametrization $\Psi: \mathcal{N} \rightarrow \mathbf{R}^{m+1}$ is given by

$$
\Psi(\vartheta)=\gamma(x) h(x)+\operatorname{grad} \gamma(x)+\vartheta, \quad x=\pi(\vartheta)
$$

where we fiberwise identify the affine relative nullity bundle over a cross section with the vector bundle $\mathcal{N}$ by parallel transport in Euclidean space.

All of the above easily extends to isometric immersions $G: N^{m} \rightarrow \mathbf{L}^{m+p}$. In particular, the Gauss parametrization is now given in terms of a submanifold $k: V^{m-\ell} \rightarrow \mathbf{H}^{m}(-1) \subset \mathbf{L}^{m+1}$ in hyperbolic space and takes the form

$$
\Upsilon(\vartheta)=\rho k-\operatorname{grad} \rho+\vartheta
$$

where $\rho \circ \pi=-\langle G, \xi\rangle$.

When $f: M^{n} \rightarrow \mathbf{R}^{n+p}, \quad p \leq n-3$, is a conformally flat submanifold, from the work of Moore ([Mo $\left.\mathbf{M o}_{1}\right]$; see also the proof below) we know that at each point there is an umbilical subspace $\mathcal{U}(x) \subset T_{x} M$ with $\operatorname{dim} \mathcal{U}(x) \geq n-p$. Hence, there is a unit vector $\eta \in T_{f(x)}^{\perp} M$ and $\mathbf{R} \ni \lambda \geq 0$, so that the second fundamental form satisfies

$$
\alpha_{f}(Z, X)=\lambda\langle Z, X\rangle \eta, \forall Z \in \mathcal{U}(x), \forall X \in T_{x} M .
$$

It is a well known fact (c.f. [Ki]) that the umbilical distribution $\mathcal{U}$ is integrable on any open subset where the index of conformal nullity $\nu_{f}^{c}(x):=\operatorname{dim} \mathcal{U}(x)$ is constant, and its leaves are extrinsic spheres in $M^{n}$ and part of round spheres in $\mathbf{R}^{n+p}$. Recall that an extrinsic sphere $\Sigma$ of a Riemannian manifold $M^{n}$ is an umbilical submanifold with parallel mean curvature vector such that the sectional curvature of $M^{n}$ is constant along planes tangent to $\Sigma$.

We say that an isometric immersion $F: N^{n+1} \rightarrow \widetilde{N}^{n+p}$ extends an isometric immersion $f: M^{n} \rightarrow \widetilde{N}^{n+p}$ when there exists an isometric embedding of $M^{n}$ into $N^{n+1}$ such that $\left.F\right|_{M}=f$. 
Theorem 2.1. Let $f: M^{n} \rightarrow \mathbf{R}^{n+p}, n \geq 5, p \leq n-3$, be a simply connected conformally flat submanifold without flat points. If $f$ has constant index of conformal nullity $\nu_{f}^{c}=\ell$, then there exist an extension $F: N^{n+1} \rightarrow \mathbf{R}^{n+p}$ of $f$ and an isometric immersion $G: N^{n+1} \rightarrow \mathbf{L}^{n+2}$ so that $M^{n}=G\left(N^{n+1}\right) \cap$ $\mathbf{V}^{n+1}$. Moreover, $F$ and $G$ carry a common $(\ell+1)$-dimensional relative nullity foliation.

Proof. We first sketch (see $\left[\mathbf{A D}_{1}\right]$ or $[\mathrm{Da}]$ for details) how one produces the isometric immersion $g: M^{n} \rightarrow \mathbf{V}^{n+1} \subset \mathbf{L}^{n+2}$ since $G$, in the statement, will be an extension of $g$ in $\mathbf{L}^{n+2}$. Take the trivial Lorentzian vector bundle $M^{n} \times \mathbf{L}^{2}$ over $M^{n}$ endowed with the compatible fiber connection which makes a canonical orthonormal basis $\{\xi, \nu\}$ parallel. Here $\|\xi\|=-1$. Now consider the symmetric bilinear form $\alpha: T M \times T M \rightarrow \mathbf{L}^{2}$ given by

$$
\alpha(X, Y)=-\left(L(X, Y)-\frac{1}{2}\langle X, Y\rangle\right) \xi+\left(L(X, Y)+\frac{1}{2}\langle X, Y\rangle\right) \nu,
$$

where $L$ is the tensor defined as

$$
L(X, Y)=\frac{1}{n-2}\left(\operatorname{Ric}(X, Y)-\frac{1}{2} n s\langle X, Y\rangle\right)
$$

and $s$ stands for the scalar curvature. Then $\alpha$ verifies the fundamental Gauss, Codazzi and Ricci equations. In fact, the Gauss equations are equivalent to the vanishing of the conformal curvature tensor (Weyl tensor), the Codazzi equations are equivalent to $L$ being a Codazzi tensor and the Ricci equations are trivially verified. We obtain from the Lorentzian version of the fundamental theorem for submanifolds that there exists an isometric immersion of $M^{n}$ into $\mathbf{L}^{n+2}$ with second fundamental form $\alpha$. Finally, in order to conclude that the immersion lies in the light cone $\mathbf{V}^{n+1}$, one has to use that the normal vector field $\xi-\nu$ is umbilic, parallel and light-like, i.e., $\|\xi-\nu\|=0$.

At each point, let $\beta: T M \times T M \rightarrow \mathbf{L}^{2} \oplus T_{f}^{\perp} M$ be the symmetric bilinear form defined by

$$
\beta(X, Y)=\left(L(X, Y)+\frac{1}{2}\langle X, Y\rangle\right) \xi+\left(L(X, Y)-\frac{1}{2}\langle X, Y\rangle\right) \nu+\alpha_{f}(X, Y) .
$$

Since $\beta$ is flat and $\beta(X, X) \neq 0$ for all $X \neq 0$, by Proposition 2 of [ $\mathbf{M o}_{1}$ ] (or Lemma 7.12 of $[\mathrm{Da}]$ ) there exist a subspace $\mathcal{U} \subset T M$, a real valued bilinear form $\phi: T M \times T M \rightarrow \mathbf{R}$ and a light-like vector $e=\xi+a \nu+b \eta$, where $\eta \in T_{f}^{\perp} M$ and $\|\eta\|=1$, such that: 
i) $\operatorname{dim} \mathcal{U} \geq n-p$,

ii) $\beta(Z, X)=\phi(Z, X) e, \forall Z \in \mathcal{U}, X \in T M$.

We conclude by a straightforward computation from $\|e\|=0$ and $i i$ ) above that there exists $\mu \in T_{g}^{\perp} M,\|\mu\|=1$, such that

$$
\alpha_{f}(Z, X)=\lambda\langle Z, X\rangle \eta, \quad \alpha_{g}(Z, X)=\lambda\langle Z, X\rangle \mu,
$$

for all $Z \in \mathcal{U}, X \in T M$.

We claim for the tangent valued second fundamental forms of $f$ and $g$ that

$$
A_{\eta}^{f}=A_{\mu}^{g}
$$

Recall that a Riemannian manifold $M^{n}$ is conformally flat if and only if its sectional curvature $K_{M}$ satisfies

$$
K_{M}(X, Y)+K_{M}(Z, W)=K_{M}(X, W)+K_{M}(Z, Y)
$$

for any orthonormal set of vectors. Using this and our assumption on $M^{n}$, it follows easily that $\lambda \neq 0$ in equations (2.2). On the other hand, we get from the Gauss equations,

$$
\lambda\left\langle\alpha_{f}(X, Y), \eta\right\rangle=\lambda\left\langle\alpha_{g}(X, Y), \mu\right\rangle, \quad \forall X, Y \in \mathcal{U}^{\perp},
$$

and the claim follows.

From the assumption that $\mathcal{U}$ has constant dimension, we have that all of the above data can be taken to be smooth. Let us denote by $\nabla$ and $\widetilde{\nabla}$ the Riemannian connections in the manifold and the ambient spaces, respectively. For any vector field $Z \in \mathcal{U}$, we have

$$
\tilde{\nabla}_{Z} Z=\nabla_{Z} Z+\lambda\langle Z, Z\rangle \eta
$$

It follows from (2.5) that

$$
L^{f}=\operatorname{span}\left\{\left(\widetilde{\nabla}_{Z} Z\right)_{\mathcal{U}^{\perp} \oplus T_{f}^{\perp} M}: \forall Z \in \mathcal{U}\right\}
$$

is a smooth line bundle over $M^{n}$. Moreover, $L^{f} \not \subset T M$ because $\lambda \neq 0$.

Since $\eta$ is parallel along $\mathcal{U}$ in the normal connection, the Codazzi equation for any normal vector field $\delta \perp \eta$ takes the form

$$
\nabla_{Z} A_{\delta}^{f} X-A_{\delta}^{f} \nabla_{Z} X+A_{\nabla_{X}^{\frac{1}{X}} \delta}^{f} Z+A_{\delta}^{f} \nabla_{X} Z=0, \quad \forall Z \in \mathcal{U} .
$$


Taking inner product with $Z$, we easily get

$$
\left\langle\widetilde{\nabla}_{Z} Z, \widetilde{\nabla}_{X} \delta\right\rangle=0 .
$$

Since $\left\langle\widetilde{\nabla}_{Z} Z, \delta\right\rangle=0$, we conclude that

$$
\widetilde{\nabla}_{X} \widetilde{\nabla}_{Z} Z \in T M \oplus \operatorname{span}\{\eta\}, \quad \forall X \in T M .
$$

For any unit vector $Z \in \mathcal{U}$, we have from (2.5) and the above that

$$
\widetilde{\nabla}_{X} \widetilde{\nabla}_{Z} Z=\nabla_{X} \nabla_{Z} Z-\lambda A_{\eta}^{f} X+\left\langle A_{\eta}^{f} \nabla_{Z} Z+\operatorname{grad} \lambda, X\right\rangle \eta,
$$

from where we easily obtain that

$$
\widetilde{\nabla}_{X}\left(\widetilde{\nabla}_{Z} Z\right)_{\mathcal{U}^{\perp}}=\nabla_{X}\left(\nabla_{Z} Z\right)_{\mathcal{U}^{\perp}}-\lambda A_{\eta}^{f} X+\left\langle A_{\eta}^{f}\left(\nabla_{Z} Z\right)_{\mathcal{U}^{\perp}}+\operatorname{grad} \lambda, X\right\rangle \eta \text {. }
$$

Let $L^{g}$ be the line bundle over $M^{n}$ similarly defined as $L^{f}$ and denote by $\tau: L^{f} \rightarrow L^{g}$ the obvious bundle isometry. Then, the maps $F: L^{f} \rightarrow \mathbf{R}^{n+p}$ and $G: L^{f} \rightarrow \mathbf{L}^{n+2}$ defined as

$$
F(\zeta)=f(x)+\zeta, \quad G(\zeta)=g(x)+\tau(\zeta), \quad x=\pi(\zeta),
$$

are immersions when restricted to a tubular neighborhood $N^{n+1}$ of the zero section. A similar calculation shows that (2.6) also holds for $g$ when $\eta$ is replaced by $\mu$. We easily conclude from (2.3) and (2.6) that $F$ and $G$ induce the same metric.

Notice that the maps $F$ and $G$ have been produced by "replacing" the extrinsic spheres by the affine subspaces of one dimension higher which contain them. This is so because the fibers of $L^{f}$ and $L^{g}$ are spanned by the mean curvature vectors of the umbilical foliation. We now show that these affine subspaces are contained in the relative nullity distributions. We have from (2.6) that the normal bundles to $F$ and $G$ are, respectively, the orthogonal complements to $\eta$ and $\mu$ in the normal bundles of $f$ and $g$. Being $\eta$ and $\mu$ parallel in the normal connection along $\mathcal{U}$, we easily see that their orthogonal complements are constant along $\mathcal{U}$. Hence, the normal bundles of $F$ and $G$ must be constant along the above affine subspaces. This concludes the proof of the theorem.

Remark 2.2. It may well happen that $M^{n}$ in Theorem 2.1 carries a foliation by $k$-dimensional extrinsic spheres with $k>\ell$. If this is the case, we have for the indices of relative nullity that $\nu_{F}=\ell+1$ but, as we show below, $\nu_{G}=k+1$. See also Proposition 4.1 for further understanding of this situation. 
From Theorem 2.1, any conformally flat hypersurface in $\mathbf{R}^{n+1}$ without flat points may always be obtained as the intersection of a flat hypersurface in $\mathbf{L}^{n+2}$ with the light cone. In fact, we have the following general result.

Theorem 2.3. Any simply connected conformally flat manifold $M^{n}$ foliated by extrinsic spheres of dimension $\ell \geq 3$ can be realized as the intersection $G\left(N^{n+1}\right) \cap \mathbf{V}^{n+1}$, where $G: N^{n+1} \rightarrow \mathbf{L}^{n+2}$ is an isometric immersion with constant index of relative nullity $\nu_{G}=\ell+1$. Conversely, any conformally flat manifold, obtained by an intersection as above, carries a foliation by $\ell$-dimensional extrinsic spheres.

Proof. We first prove the converse. In terms of a Gauss parametrization

$$
\Upsilon(\vartheta)=\rho k-\operatorname{grad} \rho+\vartheta
$$

for $G$, the intersection $g: M^{n} \rightarrow \mathbf{V}^{n+1} \subset \mathbf{L}^{n+2}$ of $G\left(N^{n+1}\right)$ with $\mathbf{V}^{n+1}$ can be parametrized as

$$
g(w)=\rho k-\operatorname{grad} \rho+\sqrt{\rho^{2}-\|\operatorname{grad} \rho\|^{2}} w=\rho(k-\mu)
$$

along the unit normal bundle $\mathcal{N}_{1}$ of $k$ in $\mathbf{H}^{n+1}(-1) \subset \mathbf{L}^{n+2}$.

All we have to show is that each fiber of $\mathcal{N}_{1}$, already part of an umbilical sphere in $\mathbf{L}^{n+1}$, is an extrinsic sphere in $M^{n}$. Taking derivatives of $\langle g, g\rangle=0$, we see that $g \in T_{g}^{\perp} M$. Thus, $T_{g}^{\perp} M=\operatorname{span}\{k, g\}$. Hence, the pair $\{k, \mu\}$ is an orthonormal basis for $T_{g}^{\perp} M$ where $\mu$ (defined by (2.7)) is normal to $M^{n}$ in $N^{n+1}$. Since $k$ and $\rho$ are constant along the leaves, we have for any $Z$ tangent to a leaf that

$$
g_{*} Z=-\rho \widetilde{\nabla}_{Z} \mu,
$$

which concludes the proof in one direction.

To prove the direct statement, let $\mathcal{U}$ denote the distribution tangent to the extrinsic spheres. Conditions (2.4) and $\ell \geq 3$ yield

$$
K_{M}(X, Y)=K_{M}(X, Z), \quad \forall Y, Z \in \mathcal{U}(x), \forall X \in \mathcal{U}^{\perp}(x) .
$$

For the tensor $L$, this implies that

$$
L(Y, Z)=\kappa\langle Y, Z\rangle, \quad \forall Y, Z \in \mathcal{U}, \quad \kappa \in C^{\infty}(M) .
$$

Moreover, using that $L$ is a Codazzi tensor, it follows easily that $\kappa$ must be constant along each extrinsic sphere. Therefore, the second fundamental form of $g: M^{n} \rightarrow \mathbf{V}^{n+1} \subset \mathbf{L}^{n+2}$ satisfies

$$
\alpha_{g}(Y, Z)=\langle Y, Z\rangle\left(\left(\frac{1}{2}-\kappa\right) \xi+\left(\frac{1}{2}+\kappa\right) \eta\right)=\langle Y, Z\rangle \delta .
$$


Hence, each extrinsic sphere is umbilic in $\mathbf{L}^{n+2}$. By the same argument that the one in the proof of Theorem 2.1, we now conclude that an extension $G$ of $g$ as required exists. Finally, observe that the condition $\nu_{G}=\ell+1$ follows from the first half of the proof.

\section{Hypersurfaces of $\mathbf{R}^{N}$ and $\mathbf{L}^{N}$.}

Our classification of Riemannian manifolds which can be simultaneously realized as hypersurfaces in both, $\mathbf{R}^{N}$ and $\mathbf{L}^{N}$, makes use of a special class of spherical and hyperbolic surfaces which we describe next.

For a spherical surface $h: V^{2} \rightarrow \mathbf{S}^{m}(1) \subset \mathbf{R}^{m+1}, m \geq 2$, together with a global coordinate system $(u, v)$, we denote by $\Gamma^{1}, \Gamma^{2}$, the two connection functions of the Riemannian connection $\nabla^{\prime}$ of $V^{2}$ determined by

$$
\nabla_{\partial_{u}}^{\prime} \partial_{v}=\Gamma^{1} \partial_{u}+\Gamma^{2} \partial_{v}
$$

where $\partial_{u}, \partial_{v}$ stand for the coordinate vector fields. Notice that $h$ is just a coordinate system when $m=2$. We say that the coordinates $(u, v)$ are conjugate whenever the second fundamental form of $h$ satisfies

$$
\alpha_{h}\left(\partial_{u}, \partial_{v}\right)=0 .
$$

In terms of the coordinate functions $\left(h^{1}, \ldots, h^{m+1}\right)$ of $h$ in $\mathbf{R}^{m+1}$, takes the form

$$
\operatorname{Hess}_{h^{j}}\left(\partial_{u}, \partial_{v}\right)+\left\langle\partial_{u}, \partial_{v}\right\rangle h^{j}=0, \quad 1 \leq j \leq m+1 .
$$

We call an associated function to a given spherical surface with conjugate coordinates $\{h,(u, v)\}$ any negative solution $\tau$ of the system of equations

$$
\left\{\begin{array}{l}
\tau_{u}=2 \Gamma^{2} \tau(1-\tau) \\
\tau_{v}=2 \Gamma^{1}(1-\tau) .
\end{array}\right.
$$

The integrability condition for system (3.3) turns out to be

$$
\left(\Gamma_{v}^{2}-2 \Gamma^{1} \Gamma^{2}\right) \tau-\Gamma_{u}^{1}+2 \Gamma^{1} \Gamma^{2}=0 .
$$

We then say that $\{h,(u, v)\}$ is a surface of first type when its metric satisfies

$$
\Gamma_{u}^{1}=\Gamma_{v}^{2}=2 \Gamma^{1} \Gamma^{2} .
$$


Surfaces of first type posses a 1-parameter family of associated functions.

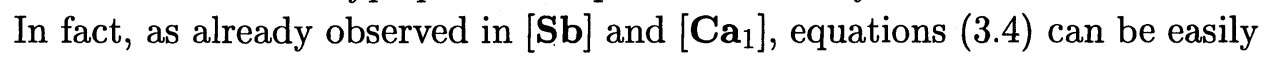
integrated. We get

$$
\Gamma^{1}=\frac{-V^{\prime}}{2(U+V)}, \quad \Gamma^{2}=\frac{-U^{\prime}}{2(U-V)},
$$

where $U=U(u)$ and $V=V(v)$ are smooth functions of one of the coordinates. Then (3.3) and (3.5) yield for the associated functions

$$
\tau=\frac{c-V(v)}{c+U(u)}
$$

where $c \in \mathbf{R}$ has to be chosen so that $\tau$ is negative.

When $\{h,(u, v)\}$ is not of first type, we call it of second type if

$$
\tau=\frac{\Gamma_{u}^{1}-2 \Gamma^{1} \Gamma^{2}}{\Gamma_{v}^{2}-2 \Gamma^{1} \Gamma^{2}}
$$

is a negative (and necessarily unique) associated function.

Remark 3.1. In their classification of isometrically deformable Euclidean

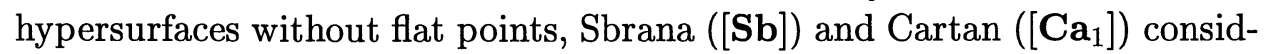
ered two classes of spherical surfaces (called by Sbrana of first and second species) which carry either real or complex conjugate coordinates. While surfaces of first type are nothing else but surfaces of first species for real conjugate coordinates, surfaces of second type are not of second species but of a similar kind. Namely, $\tau$ given by (3.6) is still a solution of system (3.3) but it is a positive one.

The notion of spherical surfaces of first or second type extends immediately to surfaces $k: V^{2} \rightarrow \mathbf{H}^{m}(-1) \subset \mathbf{L}^{m+1}$ in hyperbolic space. In this case, equation (3.2) takes the form

$$
\operatorname{Hess}_{k^{j}}\left(\partial_{u}, \partial_{v}\right)-\left\langle\partial_{u}, \partial_{v}\right\rangle k^{j}=0,1 \leq j \leq m+1 .
$$

The following is our main result in this section.

Theorem 3.2. Let $N^{m}, m \geq 2$, be a Riemannian manifold without flat points and let $F: N^{m} \rightarrow \mathbf{R}^{m+1}$ and $G: N^{m} \rightarrow \mathbf{L}^{m+1}$ be isometric immersions. Then, $F$ is locally given by the Gauss parametrization $\Psi: \mathcal{N} \rightarrow$ $\mathbf{R}^{m+1}$

$$
\Psi(\vartheta)=\gamma h+\operatorname{grad} \gamma+\vartheta
$$


in terms of a surface of first or second type $\{h,(u, v)\}$ and a solution $\gamma$ of the differential equation

$$
\operatorname{Hess}_{\gamma}\left(\partial_{u}, \partial_{v}\right)+\left\langle\partial_{u}, \partial_{v}\right\rangle \gamma=0 .
$$

Conversely, any parametrized hypersurface in $\mathbf{R}^{m+1}$ as above can be locally isometrically immersed in $\mathbf{L}^{m+1}$. A similar description holds for $G$.

Proof. From Corollary 2 of $\left[\mathrm{Mo}_{2}\right]$ and our assumption, we have everywhere for the relative nullity distributions and their indices that $\Delta_{F}=\Delta_{G}$ and $\nu_{F}=m-2=\nu_{G}$. Define $D: \Delta^{\perp} \rightarrow \Delta^{\perp}$ by

$$
D=\left(A^{F}\right)^{-1} \circ A^{G},
$$

where $A^{F}$ (respectively, $A^{G}$ ) denotes the second fundamental form of $F$ (respectively, $G$ ) restricted to $\Delta^{\perp}$. From the Gauss equations,

$$
\operatorname{det} D=-1 \text {. }
$$

Hence, for a basis $\left\{X_{1}, X_{2}\right\}$ of eigenvectors $D$ takes the form

$$
D=\left[\begin{array}{cc}
\theta & 0 \\
0 & -1 / \theta
\end{array}\right]
$$

In particular, from

$$
\left\langle A^{G} X_{1}, X_{2}\right\rangle=\left\langle A^{F} D X_{1}, X_{2}\right\rangle=\theta\left\langle A^{F} X_{1}, X_{2}\right\rangle=-\theta^{2}\left\langle A^{G} X_{1}, X_{2}\right\rangle,
$$

we conclude that $X_{1}, X_{2}$ are conjugate for $F$ and $G$, i.e.,

$$
\left\langle A^{F} X_{1}, X_{2}\right\rangle=0=\left\langle A^{G} X_{1}, X_{2}\right\rangle .
$$

We argue for $F$. We have to find necessary and sufficient conditions for the existence of a tensor field $D$ as in (3.9) so that $A^{F} \circ D$ satisfies the Codazzi equations for conjugate $X_{1}, X_{2}$. First recall that the splitting tensor $C$ assigns to each $T \in \Delta$ the endomorphism $C_{T}$ of $\Delta^{\perp}$ given by

$$
C_{T} X=-\left(\nabla_{X} T\right)_{\Delta^{\perp}} .
$$

From the Codazzi equation, we get

$$
\nabla_{T} A^{F}=A^{F} \circ C_{T}, \quad \forall T \in \Delta .
$$


Being the term on the left hand side symmetric, we have

$$
A^{F} \circ C_{T}=C_{T}^{*} \circ A^{F},
$$

where $C_{T}^{*}$ denotes the adjoint operator of $C_{T}$. Similarly, $A^{F} \circ D$ also has to satisfy (3.11). Thus,

$$
A^{F} D C_{T}=C_{T}^{*} A^{F} D=A^{F} C_{T} D .
$$

Therefore,

$$
\left[D, C_{T}\right]=0, \quad \forall T \in \Delta .
$$

On the other hand, equation (3.10) for $A^{F} \circ D$ yields

$$
A^{F} D C_{T} X=\left(\nabla_{T} A^{F}\right) D X+A^{F}\left(\nabla_{T} D\right) X, \forall X \in \Delta^{\perp}, \forall T \in \Delta .
$$

Using (3.10), we get

$$
A^{F}\left[D, C_{T}\right] X=A^{F}\left(\nabla_{T} D\right) X, \forall X \in \Delta^{\perp}, \forall T \in \Delta .
$$

We conclude from (3.12) and the above that

$$
\nabla_{T} D=0, \quad \forall T \in \Delta .
$$

Conversely, equations (3.12) and (3.13) imply that the Codazzi equation for $A^{F} \circ D$ combining vectors in $\Delta$ and $\Delta^{\perp}$ is satisfied.

Observe that equations (3.12) and (3.13) can be substituted by

$$
T(\theta)=0, \quad \forall T \in \Delta,
$$

and

$$
\nabla_{T} X_{j} \in \operatorname{span}\left\{X_{j}\right\}, \quad \forall T \in \Delta, \quad 1 \leq j \leq 2,
$$

where (3.14) says that $\theta$ is a function on $V^{2}$. Recall that $V^{2}$ is the quotient space of relative nullity leaves and that $h: V^{2} \rightarrow \mathbf{S}^{m}(1)$ an immersion induced by the Gauss map $\xi$. Moreover, condition (3.15) means that there exists a local coordinate system $(u, v)$ on $V^{2}$ such that

$$
\partial_{u} \circ \pi=\pi_{*} X_{1}, \quad \partial_{v} \circ \pi=\pi_{*} X_{2},
$$

for $X_{1}, X_{2}$ of appropriated length. In particular, this implies that $\left[X_{1}, X_{2}\right] \in$ $\Delta$. Thus, the Codazzi equation we still have to consider reduces to

$$
\nabla_{X_{1}}\left(A^{F} D X_{2}\right)=\nabla_{X_{2}}\left(A^{F} D X_{1}\right)
$$


We have,

$$
\begin{aligned}
\nabla_{X_{2}}\left(A^{F} D X_{1}\right) & =\nabla_{X_{2}}\left(A^{F} \theta X_{1}\right)=\widetilde{\nabla}_{X_{2}}\left(A^{F} \theta X_{1}\right)-\theta\left\langle A^{F} X_{1}, A^{F} X_{2}\right\rangle \xi \\
& =-\widetilde{\nabla}_{X_{2}} \xi_{*} \theta X_{1}-\theta\left\langle\xi_{*} X_{1}, \xi_{*} X_{2}\right) \xi=-\widetilde{\nabla}_{\partial_{v}} \theta \partial_{u}+\theta\left\langle\partial_{u}, \partial_{v}\right\rangle \xi \\
& =-\theta_{v} \partial_{u}-\theta\left(\nabla_{\partial_{u}} \partial_{v}+\alpha_{h}\left(\partial_{u}, \partial_{v}\right)\right) .
\end{aligned}
$$

Analogously,

$$
\nabla_{X_{1}}\left(A^{F} D X_{2}\right)=-\frac{\theta_{u}}{\theta^{2}} \partial_{v}+\frac{1}{\theta}\left(\nabla_{\partial_{u}} \partial_{v}+\alpha_{h}\left(\partial_{u}, \partial_{v}\right)\right) .
$$

Setting $\tau=-\theta^{2}$, we easily see that equation (3.16) reduces to conditions (3.1) and (3.3).

It remains to find the condition for $X_{1}, X_{2}$ to be conjugate. Recall that in terms of the Gauss parametrization,

$$
A^{F}(\vartheta)=-\left[\operatorname{Hess}_{\gamma}+\gamma \mathrm{Id}-B_{\vartheta}\right]^{-1},
$$

where Hess $\gamma$ stands for the linear operator associated to the Hessian of $\gamma$ and $B_{\vartheta}$ denotes the second fundamental form of $h$ in direction $\vartheta$. Hence, in the presence of (3.1), we have

$$
\left\langle A^{F} X_{1}, X_{2}\right\rangle=0 \Longleftrightarrow \operatorname{Hess}_{\gamma}\left(\partial_{u}, \partial_{v}\right)+\left\langle\partial_{u}, \partial_{v}\right\rangle \gamma=0 .
$$

The statement for $F$ follows from (3.1), (3.3) and (3.17), and a similar argument takes care of $G$. The converse is straightforward.

Remarks 3.3. 1) For $G$, equation (3.7) becomes

$$
\operatorname{Hess}_{\rho}\left(\partial_{u}, \partial_{v}\right)-\left\langle\partial_{u}, \partial_{v}\right\rangle \rho=0 .
$$

2) The intersection of $G\left(N^{m+1}\right)$ with a foliation of $\mathbf{L}^{m+2}$ by light cones provides a local foliation of $N^{m+1}$ by $m$-dimensional conformally flat submanifolds of $\mathbf{R}^{m+2}$. See also Theorem 1.10 of $\left[\mathbf{A D}_{1}\right]$.

3) The following special examples satisfy the assumptions of the above result:

i) $N^{m}=N^{2} \times \mathbf{R}^{m-2}$, where $N^{2}$ admits isometric immersions $F^{\prime}: N^{2} \rightarrow$ $\mathbf{R}^{3}$ and $G^{\prime}: N^{2} \rightarrow \mathbf{L}^{3}$, where $F=F^{\prime} \times$ Id and $G=G^{\prime} \times \mathrm{Id}$;

ii) $N^{m}=C N^{2} \times \mathbf{R}^{m-3}$, where $C N^{2} \cong N^{2} \times \mathbf{R}_{+}$is a cone over a surface $N^{2}$ which admits isometric immersions $F^{\prime}: N^{2} \rightarrow \mathbf{S}^{3}(1) \subset \mathbf{R}^{4}$ and $G^{\prime}: N^{2} \rightarrow \mathbf{S}_{1}^{3}(1) \subset \mathbf{L}^{4}$ into Lorentzian unit sphere, where $F=C F^{\prime} \times$ Id and $G=C G^{\prime} \times$ Id. 
We now introduce two new definitions in order to deal with the rigidity question. We call a conformally flat submanifold $f: M^{n} \rightarrow \mathbf{R}^{n+2}, n \geq$ 5 , generic when its umbilical direction $\eta \in T_{f}^{\perp} M$ (recall (2.1)) possesses everywhere a nonzero principal curvature $\lambda$ of multiplicity $n-2$. Notice that $\nu_{f}^{c}=n-2$. From (2.4) and the Gauss equations, we easily get that generic conformally flat submanifolds cannot have flat points. We say that a generic $f$ is surface-like if its isometric extension $F: N^{n+1} \rightarrow \mathbf{R}^{n+2}$ is as either one of the examples in Remark 3.3.3.

Theorem 3.4. Any local isometric deformation of a generic conformally flat submanifold $f: M^{n} \rightarrow \mathbf{R}^{n+2}, n \geq 5$, is the restriction to $M^{n}$ of an isometric deformation of its isometric extension $F: N^{n+1} \rightarrow \mathbf{R}^{n+2}$. Moreover, if nowhere surface-like, $f$ admits, precisely, a 1-parameter family of isometric deformations when $F$ is generated by a surface of first type, and it is isometrically rigid otherwise. In the surface-like situation, all deformations of $f$ are determined by isometric deformations of the surface in the first factor of $F$.

Proof. The first statement is a consequence of Theorem 5 in $\left[\mathbf{D G}_{2}\right]$. The remaining part follows from the classical Sbrana-Cartan classification of deformable Euclidean hypersurfaces $\left([\mathbf{S b}],\left[\mathbf{C a}_{1}\right]\right)$. In fact, they proved that isometric deformations of hypersurfaces splitting a surface factor or a cone of a spherical surface are given by deformations of the surface. Moreover, they also showed that hypersurfaces given by the Gauss parametrizations in terms of a surface of first species (see Remark 3.1) and a support function satisfying equation (3.7), which do not split a factor as above, admit a 1parameter family of isometric deformations. Finally, unless it splits a factor, $F$ does not belong to any class of deformable hypersurfaces when generated by a surface of second type.

Given a conformally flat submanifold $f: M^{n} \rightarrow \mathbf{R}^{n+2}$ without flat points and constant index $\nu_{f}^{c}=n-2$, it is easy to conclude from the result in [MM] that $M^{n}$ possesses two foliations by conformally flat hypersurfaces each leaf having constant $\nu^{c}=n-2$ in $\mathbf{R}^{n+2}$. Moreover, the foliation by extrinsic spheres is generate by the intersection of the two foliations. In addition, the foliations are orthogonal if and only if $f$ has flat normal bundle. Next, we present two families of examples showing that the converse to this observation, claimed in $\left[\mathbf{N o}_{1}\right]$ and $\left[\mathbf{N o}_{2}\right]$, does not hold. 
Examples 3.5. 1) Consider a surface $g^{\prime}: N^{2} \rightarrow \mathbf{L}^{3}$ which does not admit any local isometric immersion into $\mathbf{R}^{3}$. The intersection $g: N^{2} \times \mathbf{S}^{n-2}(1) \rightarrow$ $\mathbf{L}^{n+2}$ of $G=g^{\prime} \times \operatorname{Id}: N^{2} \times \mathbf{R}^{n-1} \rightarrow \mathbf{L}^{n+2}$ with $\mathbf{V}^{n+1}$, has the form

$$
g(x, v)=g^{\prime}(x)+\phi(x) v
$$

where $\phi^{2}=-\left\langle g^{\prime}, g^{\prime}\right\rangle$. Orthogonal foliations by conformally flat hypersurfaces as above, are produced by attaching the spheres to the integral curves of $\operatorname{grad} \phi$ and $(\operatorname{grad} \phi)^{\perp}$. It follows from Theorem 3.2 that $N^{2} \times \mathrm{S}^{n-2}(1)$ cannot be isometrically immersed in $\mathbf{R}^{n+2}$ with the metric induced by $g$.

2) Take any surface $k: N^{2} \rightarrow \mathbf{H}^{n+1}(-1)$ with flat normal bundle and principal coordinates $(u, v)$ which is not of first or second type. Let $G: N^{n+1} \rightarrow \mathbf{L}^{n+2}$ be the hypersurface defined in terms of the Gauss parametrization by $k$ and the support function $\rho=1$. The intersection $g: N^{2} \times \mathbf{S}^{n-2}(1) \rightarrow \mathbf{L}^{n+2}$ of $G\left(N^{n+1}\right)$ with $\mathbf{V}^{n+1}$ is of the form

$$
g\left(u, v, t_{1}, \ldots, t_{n-2}\right)=k(u, v)+\sum_{j=1}^{n-1} \psi_{j}\left(t_{1}, \ldots, t_{n-2}\right) \xi_{j}(u, v),
$$

where $\xi_{1}, \ldots, \xi_{n-1}$ is a parallel orthonormal normal frame for $k$ and $\psi=$ $\left(\psi_{1}, \ldots, \psi_{n-1}\right)$ a parametrization of the unit sphere in $\mathbf{R}^{n-1}$. A straightforward calculation shows that the foliations obtained fixing values of $u$ or $v$ verify all of the above conditions but $N^{2} \times \mathbf{S}^{n-2}(1)$ cannot be isometrically immersed in $\mathbf{R}^{n+2}$ with the metric induced by $g$.

\section{The parametrization.}

In this section we provide an explicit parametrization for any nonflat conformally flat submanifolds in codimension 2 .

That $f: M^{n} \rightarrow \mathbf{R}^{n+2}$ is a composition we mean that there exist an open subset $U \subset \mathbf{R}^{n+1}$ and isometric immersions $\tilde{f}: M^{n} \rightarrow U$ and $H: U \rightarrow$ $\mathbf{R}^{n+2}$ such that $f=H \circ \tilde{f}$.

Proposition 4.1. Any conformally flat submanifold $f: M^{n} \rightarrow \mathbf{R}^{n+2}, n \geq$ 5 , without flat points is locally, along an open dense subset either generic or a composition.

Proof. Since we are dealing with a local statement, we may assume that the principal curvature $\lambda \neq 0$ of the umbilical direction $\eta$ (see (2.1)) has constant 
multiplicity $\ell$. When $\ell \geq n-1$, we have from (2.4) that $\operatorname{rank} A_{\xi} \leq 1$ for unit $\xi \perp \eta$. On any open subset of $M^{n}$ where $A_{\xi}$ has constant rank, we verify by a straightforward computation that $A_{\eta}$ satisfies the Gauss and Codazzi equations for a hypersurface $\tilde{f}: M^{n} \rightarrow \mathbf{R}^{n+1}$. It follows from Theorem 8 of $\left[\mathbf{D T}_{1}\right]$ that $f$ is a composition.

Notice that compositions as in the above result can easily be described parametrically by using together the Gauss parametrization and Cartan's parametrization of conformally flat hypersurfaces referred to in the introduction. We now consider the generic case.

Theorem 4.2. Let $h: V^{2} \rightarrow \mathbf{S}^{n+1}(1) \subset \mathbf{R}^{n+2}, n \geq 2$, be a surface of first or second type with conjugate coordinates $(u, v)$. For a given associated function $\tau$, let $\Theta^{*}$ be the adjoint to the tensor $\Theta: T V \rightarrow T V$ defined by

$$
\Theta \partial_{u}=\frac{1}{\theta} \partial_{u}, \quad \Theta \partial_{v}=-\theta \partial_{v}
$$

where $\theta=\sqrt{-\tau}$. Moreover, let $\rho$ be a solution of the differential equation

$$
\rho_{u v}+\theta^{2} \Gamma^{2} \rho_{v}+\frac{1}{\theta^{2}} \Gamma^{1} \rho_{u}+\rho\left\langle\partial_{u}, \partial_{v}\right\rangle=0
$$

and let $\beta: V^{2} \rightarrow \mathbf{R}^{n+2}$ be a solution, unique up to translations, of the completely integrable system of first order

$$
\left\{\begin{array}{l}
\beta_{u}=\theta \rho h_{u}-\frac{\rho_{u}}{\theta} h \\
\beta_{v}=-\frac{\rho}{\theta} h_{v}+\theta \rho_{v} h .
\end{array}\right.
$$

Then, on the open subset of regular points, the map $\varphi: \mathcal{N}_{1} \rightarrow \mathbf{R}^{n+2}$, defined on the unit normal bundle $\mathcal{N}_{1}$ of $h$ in the sphere and given by

$$
\varphi(w)=\beta-\Theta^{*} \operatorname{grad} \rho+\sqrt{\rho^{2}-\left\|\Theta^{*} \operatorname{grad} \rho\right\|^{2}} w
$$

is a parametrization of a generic n-dimensional conformally flat submanifold of $\mathbf{R}^{n+2}$. Conversely, for $n \geq 5$, any generic conformally flat submanifold $f: M^{n} \rightarrow \mathbf{R}^{n+2}$ can be locally parametrized this way.

Proof. We prove the converse. Consider a local isometric immersion of $M^{n}$ into the light cone $g: M^{n} \rightarrow \mathbf{V}^{n+1} \subset \mathbf{L}^{n+2}$, and the isometric extensions $F: N^{n+1} \rightarrow \mathbf{R}^{n+2}$ of $f$ and $G: N^{n+1} \rightarrow \mathbf{L}^{n+2}$ of $g$. From the proofs of 
Theorem 2.1 and Proposition 2.3, there exist orthonormal bases $\{h, \eta\}$ of $T_{f}^{\perp} M$ and $\{k, \mu\}$ of $T_{g}^{\perp} M$, where $h, k$ are seen simultaneously as Gauss maps for $F$ and $G$ and as certain immersions $h: V^{2} \rightarrow \mathbf{S}^{n+1}(1)$ and $k: V^{2} \rightarrow$ $\mathbf{H}^{n+1}(-1)$. Equation (3.8) yields

$$
A_{k}^{g}=A_{h}^{f} \circ D
$$

for $D$ extended to $T M$ as $\left.D\right|_{\mathcal{U}}=0$. On the other hand, we easily get taking derivatives of (2.7) and using (2.3) that

$$
\operatorname{Id}=\rho\left(A_{\eta}^{f}-A_{k}^{g}\right),
$$

where $\rho=1 / \lambda$ (see (2.1)) is considered as a function on $V^{2}$. Therefore,

$$
\rho A_{h}^{f} \circ D=\rho A_{\eta}^{f}-\mathrm{Id} .
$$

Consider the map $\beta: M^{n} \rightarrow \mathbf{R}^{n+2}$, defined as

$$
\beta=f+\rho \eta \text {. }
$$

Then, $\beta$ can also be viewed as defined along $V^{2}$ since

$$
\beta_{*} Z=f_{*} Z+\rho \widetilde{\nabla}_{Z} \eta=Z-\rho A_{\eta}^{f} Z=0, \quad \forall Z \in \mathcal{U} .
$$

Our purpose is to describe $f$ parametrically as

$$
f=\beta-\rho \eta,
$$

where $\beta$ and $\eta$ are expressed in terms of data generated by $h$. Since the images by $f$ of the leaves of $\mathcal{U}$ are spheres, we have from (4.5) that $\eta$, when restricted to a leaf, parametrizes part of a round $(n-2)$-dimensional sphere contained in some $(n-1)$-dimensional affine subspace of $\mathbf{R}^{n+2}$. Fix a leaf $L$ of $\mathcal{U}, x_{0} \in L, Y \in \mathcal{U}^{\perp}\left(x_{0}\right)$ and set $y=\pi_{*} Y$. For any $Z \in T L$, we have

$$
Z\left\langle\eta, h_{*} y\right\rangle=Z\left\langle\eta, h_{*} \pi_{*} Y\right\rangle=\left\langle\tilde{\nabla}_{Z} \eta, h_{*} \pi_{*} Y\right\rangle=\left\langle A_{\eta}^{f} Z, A_{h}^{f} Y\right\rangle=\frac{1}{\rho}\left\langle A_{h}^{f} Z, Y\right\rangle=0 .
$$

Therefore, the $h_{*}\left(T_{\pi\left(x_{0}\right)} V\right)$-component of $\left.\eta\right|_{L}$ is constant. It follows easily that there exists $\sigma \in T V$ so that $\eta$ can be written as

$$
\eta=h_{*} \sigma+\Phi+\kappa \psi
$$

where $\Phi \in \mathcal{N} \subset T S^{n+1}(1), \kappa \in C^{\infty}(V)$ and $\psi(x)$ is a parametrization of the unit sphere in the fiber $\mathcal{N}([x])$ when $x$ varies along $L$. Using $\|\eta\|=1$, we easily obtain that $\Phi=0$. Hence,

$$
\eta=h_{*} \sigma-\sqrt{1-\left\|h_{*} \sigma\right\|^{2}} \psi .
$$


Let $\phi(x): \mathcal{U}^{\perp}(x) \rightarrow T_{[x]} V$ be the isomorphism $\phi(x)=\left.\pi_{*}\right|_{\mathcal{U}^{\perp}(x)}$. From (4.4), we have for $X \in \mathcal{U}^{\perp}(x)$,

$$
\begin{aligned}
\beta_{*} X & =X+\rho \widetilde{\nabla}_{X} \eta+X(\rho) \eta \\
& =\left(\operatorname{Id}-\rho A_{\eta}^{f}\right) X-\rho\left\langle\widetilde{\nabla}_{X} h, \eta\right\rangle h+X(\rho) \eta \\
& =-\rho A_{h}^{f} D X-\rho\left\langle\widetilde{\nabla}_{X} h, \eta\right\rangle h+X(\rho) \eta .
\end{aligned}
$$

Since $h, \rho$ and $\beta$ can be viewed as maps on $V^{2}$, the above and (4.6) yield

$$
\beta_{*} \phi X=\rho h_{*} \phi D X-\rho\langle\phi X, \sigma\rangle h+(\phi X(\rho)-\rho\langle\phi D X, \sigma\rangle) \eta .
$$

By (3.13), a tensor field $\Omega$ on $V^{2}$ is defined so that

$$
\Omega \circ \phi=\phi \circ D \text {. }
$$

Notice that $\Theta=\Omega^{-1}$. Only $\eta$ in (4.7) is not constant along the leaves of $\mathcal{U}$. Hence, this equation is equivalent to

$$
\sigma=\frac{1}{\rho} \Theta^{*} \operatorname{grad} \rho
$$

and

$$
\beta_{*} y=\rho h_{*} \Theta^{-1} y-\langle\Theta y, \operatorname{grad} \rho\rangle h, \forall y \in T V .
$$

In conjugate coordinates, (4.9) takes the form

$$
\left\{\begin{array}{l}
\beta_{u}=\rho \theta h_{u}-\frac{\rho_{u}}{\theta} h \\
\beta_{v}=-\frac{\rho}{\theta} h_{v}+\theta \rho_{v} h .
\end{array}\right.
$$

Set $\tau=-\theta^{2}$. An easy computation shows that equations (3.1), (3.3) and (4.1) are the integrability conditions for (4.10). Therefore, system (4.10) is completely integrable if and only if $h$ is a surface of first or second type and $\rho$ verifies (4.1). The proof follows from (4.5), (4.6), (4.8) and (4.10). The direct statement is straightforward.

Remarks 4.3. 1) A long but straightforward calculation using (4.3) and that $f$ and $g$ are isometric yields

$$
\langle x, y\rangle^{\prime}=\left\langle\Theta^{-1} x, \Theta^{-1} y\right\rangle, \quad \forall x, y \in T V,
$$


where we denote by $\langle,\rangle^{\prime}$ and $\nabla^{\prime \prime}$ the metric and connection induced on $V^{2}$ by $k$. Therefore,

$$
\nabla_{x}^{\prime \prime} y=\Theta \nabla_{x}^{\prime} \Theta^{-1} y
$$

Then,

$$
\Theta^{-1} \operatorname{grad}^{\prime} \rho=\Theta^{*} \operatorname{grad} \rho
$$

and

$$
\operatorname{Hess}_{\rho}^{\prime}\left(\partial_{u}, \partial_{v}\right)-\rho\left\langle\partial_{u}, \partial_{v}\right\rangle^{\prime}=\frac{1}{\theta}\left\{\rho_{u v}+\theta^{2} \Gamma^{2} \rho_{v}+\frac{1}{\theta^{2}} \Gamma^{1} \rho_{u}+\rho\left\langle\partial_{u}, \partial_{v}\right\rangle\right\} .
$$

Hence, equations (3.18) and (4.1) are equivalent. Also, the radius of the umbilical spheres in both ambient spaces (c.f. (2.7) and (4.2)) are equal, as they must be, since $f$ and $g$ are isometric, that is,

$$
s:=\sqrt{\rho^{2}-\left\|\Theta^{*} \operatorname{grad} \rho\right\|^{2}}=\sqrt{\rho^{2}-\left\|\operatorname{grad}^{\prime} \rho\right\|^{\prime 2}} .
$$

2) It is easy to see that the regular points of the Euclidean parametrization are the ones for which the operator $P-s B_{w}$ is nonsingular. Here $P$ is defined as

$$
\langle P x, y\rangle=\left\langle\rho \Theta^{-1} x-\nabla_{x}^{\prime}\left(\Theta^{*} \operatorname{grad} \rho\right), y\right\rangle,
$$

and $B_{w}$ stands for the second fundamental form of $h$ in $\mathbf{S}^{n+1}(1)$ for the normal direction $w$.

We conclude the section presenting a large family of examples generated by a class of spherical surfaces of first type already considered by Cartan $\left(\left[\mathbf{C a}_{1}\right]\right)$.

Examples 4.4. Given a spherical surface $h: V^{2} \rightarrow \mathbf{S}^{n+1}(1) \subset \mathbf{R}^{n+2}$ of first type, define $H: V^{2} \rightarrow \mathbf{R}^{n+2}$ by $H=\sqrt{U+V} h$. From (3.2) and (3.5) we have that, componentwise, any surface of first type is produced giving a set of functions $\left(h^{1}, \ldots, h^{m+1}\right)$, with $\sum_{j=1}^{m+1}\left(h^{j}\right)^{2}=1$, all satisfying the same differential equation:

$$
h_{u v}^{j}+\frac{V^{\prime}}{2(U+V)} h_{u}^{j}+\frac{U^{\prime}}{2(U+V)} h_{v}^{j}+F h^{j}=0, \quad 1 \leq j \leq m+1,
$$

where $U=U(u), V=V(v)$ and $F=F(u, v)$ are arbitrary smooth functions. A straightforward computation shows that equation (4.11) takes the form

$$
H_{u v}+\left(\Gamma^{1} \Gamma^{2}+F\right) H=0, \quad F=\left\langle\partial_{u}, \partial_{v}\right\rangle .
$$


Let us analyze the case when

$$
\Gamma^{1} \Gamma^{2}+F=0 .
$$

In this situation, there exist two regular curves $\alpha_{j}: I_{j} \rightarrow \mathbf{R}^{n+2}$ so that

$$
H(u, v)=\alpha_{1}(u)+\alpha_{2}(v) .
$$

Hence,

$$
U(u)+V(v)=\left\|\alpha_{1}\right\|^{2}+\left\|\alpha_{2}\right\|^{2}+2\left\langle\alpha_{1}, \alpha_{2}\right\rangle .
$$

In particular, $\left\langle\alpha_{1}^{\prime}(u), \alpha_{2}^{\prime}(v)\right\rangle=0$ along $I_{1} \times I_{2}$. Therefore, there are affine orthogonal subspaces $E_{j} \subset \mathbf{R}^{n+2}$ such that $\alpha_{j}\left(I_{j}\right) \subset E_{j}, 1 \leq j \leq 2$, and

$$
U(u)=\left\|\alpha_{1}\right\|^{2}, V(v)=\left\|\alpha_{2}\right\|^{2} .
$$

Hence, surfaces of first type satisfying the additional condition (4.12) have the expression

$$
h(u, v)=\frac{\left(\alpha_{1}(u), \alpha_{2}(v)\right)}{\sqrt{\left\|\alpha_{1}(u)\right\|^{2}+\left\|\alpha_{2}(v)\right\|^{2}}} .
$$

Assume, in addition, that the $\alpha_{j}$ 's are spherical curves, i.e., $\left\|\alpha_{j}\right\|=c_{j}$, $c_{j} \in \mathbf{R}_{+}$with $c_{1}^{2}+c_{2}^{2}=1$. Then, $F=\Gamma^{1}=\Gamma^{2}=0, \tau=-k^{2}$ is constant and

$$
\rho(u, v)=\rho_{1}(u)+\rho_{2}(v) .
$$

We conclude that

$$
\begin{aligned}
\varphi(w)= & \frac{1}{k}\left(k^{2} \rho \alpha_{1}-\left(1+k^{2}\right) \int \rho_{1}^{\prime} \alpha_{1} d u,-\rho \alpha_{2}-\left(1+k^{2}\right) \int \rho_{2}^{\prime} \alpha_{2} d v\right) \\
& +\phi+\sqrt{\rho^{2}-\|\phi\|^{2}} w,
\end{aligned}
$$

where

$$
\phi=\left(\frac{\rho_{1}^{\prime} \alpha_{1}^{\prime}}{k\left\|\alpha_{1}^{\prime}\right\|^{2}},-\frac{k \rho_{2}^{\prime} \alpha_{2}^{\prime}}{\left\|\alpha_{2}^{\prime}\right\|^{2}}\right)
$$

parametrizes a 1-parameter family of generic conformally flat submanifolds.

The above set of examples contains very simple ones obtained by taking $\rho=1$. This yields a 1 -parameter family of immersions of the unit normal bundle $\mathcal{N}_{1}$ of $h$ in $\mathbf{S}^{n+1}(1)$, given by

$$
\varphi(w)=\left(k \alpha_{1},-\frac{1}{k} \alpha_{2}\right)+w, \quad k \in \mathbf{R}_{+} .
$$

These examples are even simpler if the $\alpha_{j}$ 's are taken to be circles. 


\section{The flat case.}

Our goal in this section is to parametrically describe all flat Euclidean submanifolds in codimension 2 which cannot be obtained as compositions. Arguments here will be quite sketchy in regard to their similarity with the ones in [CD].

We assume that $f: M^{n} \rightarrow \mathbf{R}^{n+2}$ is flat, 1-regular and nowhere a composition. The first assumption means that the first normal spaces $N_{1}^{f}(x) \subset T_{f(x)}^{\perp} M$, i.e., the subspaces spanned by the second fundamental form at each point, form a subbundle of $T_{f}^{\perp} M$. By Theorem 1 in [DT $\left.\mathbf{D T}_{2}\right]$ we have that $\operatorname{dim} N_{1}^{f}=2$. As in [CD], we conclude that in a neighborhood of each point there exists an orthonormal normal local basis $\{\xi, \eta\}$ for globally defined line bundles in $T_{f}^{\perp} M$, such that

$$
\operatorname{rank} A_{\xi}=\operatorname{rank} A_{\eta}=1
$$

and, on an open dense subset,

$$
\operatorname{ker} A_{\xi} \not \subset \operatorname{ker} \psi, \operatorname{ker} A_{\eta} \not \subset \operatorname{ker} \psi,
$$

where $\psi$ is the 1 -form defined as

$$
\psi^{\prime}(X)=\left\langle\nabla_{X}^{\perp} \xi, \eta\right\rangle .
$$

We say that a 1-regular flat submanifold $f: M^{n} \rightarrow \mathbf{R}^{n+2}$ is generic when $\operatorname{dim} N_{1}^{f}=2$ and condition (5.1) holds everywhere. So $f$ is nowhere a composition.

By Codazzi's equation,

$$
A_{\xi}[X, W]=-\psi(X) A_{\eta} W, \quad \forall X \in \Delta,
$$

where $0 \neq W \in \operatorname{ker} A_{\xi} \cap \Delta^{\perp}$. From $\operatorname{Im} A_{\xi} \neq \operatorname{Im} A_{\eta}$ and $A_{\eta} W \neq 0$, we get

$$
\Delta \subset \operatorname{ker} \psi \text {. }
$$

It follows easily from $\operatorname{ker} A_{\xi} \not \subset \operatorname{ker} \psi$ that $h: V^{2} \rightarrow \mathbf{S}^{n+1}(1)$, defined by $\xi=h \circ \pi$, is an immersion. Here, $V^{2}$ is the quotient space of leaves of relative nullity of $M^{n}$ with projection $\pi: M^{n} \rightarrow V^{2}$ endowed with the metric induced by $h$.

For all $X \in \Delta$ and $Y \in T M$, we have that

$$
0=\left\langle A_{\xi} X, Y\right\rangle=-\left\langle\xi_{*} Y, f_{*} X\right\rangle .
$$


Hence, $\Delta(x) \subset T_{h([x])}^{\perp} V$. Set $\tilde{\gamma}=\langle f, \xi\rangle$. Then,

$$
X(\tilde{\gamma})=\left\langle f, \xi_{*} X\right\rangle=0, \quad \forall X \in \Delta .
$$

Therefore, $\tilde{\gamma}$ is constant along the leaves of $\Delta$. Let $\delta \in T_{h}^{\perp} V$ be a unit vector field orthogonal to $\Delta$. Similar to Proposition 3.5 of $[\mathbf{C D}]$, we can define a parametrization $\varphi$ of $f$ along the subbundle $N_{\delta}=\left\{\beta \in T_{h}^{\perp} V:\langle\beta, \delta\rangle=0\right\}$ by

$$
\varphi(\beta)=(\gamma h+\operatorname{grad} \gamma+\theta \delta)(y)+\beta, \quad y=\pi(\beta),
$$

where $\gamma$ is defined by $\tilde{\gamma}=\gamma \circ \pi$. Notice that $\theta \in C^{\infty}(V)$ since $f_{*} X(x)=$ $X(x) \in N_{\delta}([x])$ for all $X \in \Delta$.

Let $P$ be the symmetric tensor field on $V^{2}$ defined as $P=$ Hess $_{\gamma}+\gamma$ Id. We have that $\varphi_{*} Y=Y$ for any $Y \in N_{\delta}(\pi(\beta))$ in the fiber at $\pi(\beta) \in V^{2}$. If $T \in T_{\beta} N_{\delta}$ with $\pi_{*} T=z$, a calculation similar to that of Proposition 3.9 in $[\mathrm{CD}]$ gives

$$
\varphi_{*} T(\beta)=h_{*}\left(P-\theta B_{\delta}-B_{\beta}\right) z+\left(\left\langle B_{\delta} \operatorname{grad} \gamma+\operatorname{grad} \theta, z\right\rangle-\left\langle\nabla_{z}^{\perp} \delta, \beta\right\rangle\right) \delta+\mu
$$

where $\mu \in N_{\delta}(\pi(\beta))$ and $B_{\delta}$ denotes the second fundamental form of $h$ in the normal direction $\delta$. Set $\phi=\left.\pi_{*}\right|_{\Delta^{\perp}}$. From $W \in \operatorname{ker} A_{\xi}$, we get $\eta=\lambda \xi_{*} W=h_{*} \phi \lambda W$, for some function $\lambda \neq 0$. Since $\eta$ is constant along the leaves of relative nullity, it follows that there exists a unit vector field $w \in T V$ such that

$$
\eta=h_{*} w \circ \pi
$$

By (5.4), this is equivalent to

$$
B_{\beta} w=0, \forall \beta \in N_{\delta}, \text { and }\left(P-\theta B_{\delta}\right) w=0 .
$$

But,

$$
A_{\eta} \lambda W=\lambda^{2} A_{\xi_{*} W} W=\left\langle B_{\delta} w, w\right\rangle \delta \circ \pi+\epsilon W^{\perp}, \epsilon \in C^{\infty}(V),
$$

where $W^{\perp}$ is a unit generator of $\operatorname{Im} A_{\xi}$. From $\operatorname{dim} N_{f}^{1}=2$, we conclude that

$$
\left\langle B_{\delta} w, w\right\rangle \neq 0 \text {. }
$$

Since $\operatorname{rank} A_{\eta}=1$ and $\operatorname{dim} N_{f}^{1}=2$, we have that there exists a vector field $V \in \Delta^{\perp}$, linearly independent with $W$, such that

$$
A_{\xi_{*} W} V=0 .
$$


As in [CD], using (5.4) and (5.5), we easily conclude that there exists a vector field $v \in T V$, linearly independent with $w(v \circ \pi=\phi V)$, verifying

$$
\nabla_{v}^{\prime} w=0, \quad \alpha_{h}(v, w)=0 .
$$

By (5.8), equations (5.6) are equivalent to

$$
\delta=\frac{\alpha_{h}(w, w)}{\left\|\alpha_{h}(w, w)\right\|}, \quad \theta=\frac{\langle P w, w\rangle}{\left\|\alpha_{h}(w, w)\right\|} \quad \text { and } \quad\langle P v, w\rangle=0 .
$$

Extending the definition in [CD], we call $h: V^{2} \rightarrow \mathbf{S}^{n+1}(1)$ a surface of type $C$ when there exists a conjugate coordinate system $(u, v)$ such that

$$
\Gamma^{1}=0 \text { and } \alpha_{h}\left(\partial_{v}, \partial_{v}\right) \neq 0 .
$$

Theorem 5.1. Let $h: V^{2} \rightarrow \mathbf{S}^{n+1}(1)$ be a surface of type $C$, and let $\gamma$ be any solution of equation

$$
\operatorname{Hess}_{\gamma}\left(\partial_{u}, \partial_{v}\right)+\left\langle\partial_{u}, \partial_{v}\right\rangle \gamma=0 .
$$

Let $\delta \in T_{h}^{\perp} V$ and $\theta \in C^{\infty}(V)$ be given by

$$
\delta=\frac{\alpha_{h}\left(\partial_{v}, \partial_{v}\right)}{\left\|\alpha_{h}\left(\partial_{v}, \partial_{v}\right)\right\|}, \quad \theta=\frac{\left\langle P \partial_{v}, \partial_{v}\right\rangle}{\left\|\alpha_{h}\left(\partial_{v}, \partial_{v}\right)\right\|} .
$$

Then, on the open subset of regular points, the map $\varphi: N_{\delta} \rightarrow \mathbf{R}^{n+2}$, defined on $N_{\delta}=\left\{\beta \in T_{h}^{\perp} V:\langle\delta, \beta\rangle=0\right\}$ and given by

$$
\varphi(\beta)=\gamma h+\operatorname{grad} \gamma+\theta \delta+\beta,
$$

is a parametrization of a generic $n$-dimensional flat submanifold of $\mathbf{R}^{n+2}$. Conversely, any such submanifold can be locally parametrized this way.

Proof. Follows using (5.3), (5.7), (5.8) and (5.9).

Remarks 5.2. 1) The regular points of $\varphi$ are characterized by conditions:

$$
\left\langle B_{\delta} \operatorname{grad} \gamma+\operatorname{grad} \theta, \partial_{v}\right\rangle \neq\left\langle\nabla \frac{1}{\partial_{v}} \delta, \beta\right\rangle, \quad\left\langle\left(P-\theta B_{\delta}-B_{\beta}\right) \partial_{u}, \partial_{u}\right\rangle \neq 0 .
$$

2) As in [CD], we have that $R_{f}^{\perp}=0 \Longleftrightarrow \nabla_{w}^{\prime} w=0$. 


\section{References.}

[AD $\mathbf{A D}_{1}$ A. Asperti and M. Dajczer, Conformally flat Riemannian manifolds as hypersurfaces of the light cone, Canadian Math. Bull. 32 (1989), 281-285.

$\left[\mathbf{A D}_{2}\right] \quad$ A. Asperti and M. Dajczer, $N$-dimensional submanifolds of $R^{N+1}$ and $S^{N+2}$, Illinois J. of Math. 28 (1984), 621-645.

[CD] M. do Carmo and M. Dajczer, Local isometric immersions of $R^{2}$ into $R^{4}$, J. reine angew. Math. 442 (1993), 205-219.

[Ca $\left.\mathbf{C a}_{1}\right]$ E. Cartan, La déformation des hypersurfaces dans l'espace euclidien réel a $n$ dimensions, Bull. Soc. Math. France 44 (1916), 65-99.

[Ca $\left.\mathbf{C a}_{2}\right]$ E. Cartan, Sur certains hypersurfaces de l'espace conforme réel a cinq dimensions, Bull. Soc. Math. France 46 (1918), 84-105.

[CDM] M. do Carmo, M. Dajczer and F. Mercuri, Compact conformally flat hypersurfaces, Trans. Amer. Math. Soc. 288 (1985), 189-203.

[CY] B. Chen and Y. Yano, Special conformally flat spaces and canal hypersurfaces, Tôhoku Mat. J. 25 (1973), 177-184.

[Da] M. Dajczer et all, "Submanifolds and isometric immersions". Math. Lec. Series 13, Publish or Perish Inc. Houston, 1990.

[DFT] M. Dajczer, L. Florit and R. Tojeiro, On deformable hypersurfaces in space forms, Preprint.

[DG $\mathbf{D G}_{1}$ M. Dajczer and D. Gromoll, Gauss parametrizations and rigidity aspects of submanifolds, J. Differential Geometry 22 (1985), 1-12.

$\left[\mathbf{D G}_{2}\right]$ M. Dajczer and D. Gromoll, Isometric deformations of compact Euclidean submanifolds in codimension 2, Duke Math. J. 79 (1995), 605-618.

[DT I $_{1}$ M. Dajczer and R. Tojeiro, On compositions of isometric immersions, J. Diff. Geometry 36 (1992), 1-18.

[DT $\mathbf{D T}_{2}$ M. Dajczer and R. Tojeiro, Submanifolds with nonparallel first normal bundle, Canad. Math. Bull. 37 (1994), 330-337.

[Ki] Y. Kitagawa, Umbilics of conformally flat submanifolds, Tôhoku Math. J. 32 (1980), 433-438.

[Mo $\left.\mathbf{M o}_{1}\right]$ J. Moore, Conformally flat submanifolds of Euclidean space, Math. Ann. 225 (1977), 89-97. 
[Mo $\left.\mathrm{Mo}_{2}\right]$ J. Moore, Submanifolds of constant positive curvature I, Duke Math. J. 44 (1977), 449-484.

[MM] J. Moore and J. Morvan, Sous-varieétés conformément plates de codimension quatre, C. R. Acad. Sc. Paris 287 (1978), 655-657.

[MZ] M. Morvan and G. Zafindratafa, Conformally flat submanifolds, Ann. fac. sci. Toulouse 8 (1986), 331-347.

[No $\mathbf{N o}_{1}$ M. Noronha, Conformally flat immersions in codimension two, Geometriae Dedicata 23 (1987), 115-130.

[No $\mathrm{No}_{2}$ M. Noronha, Conformally flat immersions and flatness of the normal connection, Pacific J. of Math. 138 (1989), 145-150.

[Sb] V. Sbrana, Sulla varietà ad $n-1$ dimensioni deformabili nello spazio euclideo ad $n$ dimensioni, Rend. Circ. Mat. Palermo 27 (1909), 1-45.

RECEIVED MARCH 29TH, 1995.

IMPA

Est. DONA Castorina 110

22460-320 - RiO DE JANEIRO - BRAZIL.

E-MAIL ADDRESSES: MARCOS@IMPA.BR, LUIS@IMPA.BR 